\title{
Correction to: Manipulation of bio-micro/nanoparticles in non-Newtonian microflows
}

\author{
Fei Tian ${ }^{1,2} \cdot$ Qiang Feng ${ }^{2} \cdot$ Qinghua Chen ${ }^{2} \cdot$ Chao $\mathrm{Liu}^{2,3} \cdot{\text { Tiejun } \mathrm{Li}^{1} \cdot \text { Jiashu Sun }}^{2,4}$ (I) \\ Published online: 7 May 2019 \\ ○) Springer-Verlag GmbH Germany, part of Springer Nature 2019
}

\section{Correction to: Microfluid Nanofluid (2019) 23:68 https://doi.org/10.1007/s10404-019-2232-z}

In the original publication, names of three authors were incorrect.

The correct author names are as below,

Fei Tian, Qiang Feng, Qinghua Chen, Chao Liu, Tiejun Li, Jiashu Sun

The original article has been corrected.

The original article can be found online at https://doi.org/10.1007/ s10404-019-2232-z.

Chao Liu

liuc@nanoctr.cn

$\triangle$ Tiejun Li

li_tiejun@hebut.edu.cn

$\bowtie$ Jiashu Sun

sunjs@nanoctr.cn

1 School of Mechanical Engineering, Hebei University of Technology, Tianjin 300401, China

2 CAS Key Laboratory of Standardization and Measurement for Nanotechnology, CAS Center for Excellence in Nanoscience, National Center for Nanoscience and Technology, Beijing 100190, China

3 School of Mechanical Engineering, and Jiangsu Key Laboratory for Design and Manufacture of Micro-Nano Biomedical Instruments, Southeast University, Nanjing 211189, China

4 University of Chinese Academy of Sciences, Beijing 100049, China 